\title{
PASSING FROM A GAS TO AN ELECTRIC WATER HEATER SYSTEM: ADAPTABLE PID VERSUS SMITH PREDICTIVE CONTROL
}

\author{
José Vieira and Alexandre Mota
}

\begin{abstract}
This paper presents the control results of an electric water heater system using two approaches: adaptable proportional integral derivative and Smith predictive control based in the physical internal model control structure.

The electric water heater was modelled with two variable blocks connected in series: a first order system and a time delay. In fact, the gain, the time constant and the time delay of the system change linearly with the water that flows in the permutation chamber. The physical model of the electric water heater system was retched based in energy dynamic equations and validated with open loop data of the system in a similar way that was made in a previews study about modelling and controlling a gas water heater.

The two different control algorithms explored are the adaptive proportional integral derivative (APID) and the Smith predictive control (SPC) based in the internal physical model control algorithm. The first approach has some problems dealing with the time constant and the time delay variations of the system. This solution can control the overshoot for all different water flows but the time constant of the close loop systems changes with the water flow. The APID does not deal well with water flow variations. The second approach is more adequate to control this kind of systems (first order system followed by a time delay that changes in time). The SPC loop is indicated for control time delay systems and with the à priori knowledge of the physical model we can achieve a very good control result.

Finally, these two algorithms are applied in controlling the system and the results are compared using the mean square error criterion.
\end{abstract}

Index terms - adaptive PID, electrical water heater, physical model identification, Smith predictive control and time delay system.

\section{INTRODUCTION}

Industry control processes presents many challenging problems, including non-linear or variable linear dynamic behaviour, variable time delay that means time varying parameters. One of the alternatives to handle with time delay systems is to use prediction technique to compensate the negative influence of the time delay. Smith predictor control

\footnotetext{
J. A. Vieira is with Department of Electrotecnica of the Escola Superior de Tecnologia, Av. Empresário, 600-767 Castelo Branco, Portugal (corresponding author phone: 272 339300; fax: 272 339399; email: zevieira@est.ipcb.pt).

A. M. Mota is with Department of Electrónica, Telecomunicações e Informatica of the Universidade de Aveiro, Campus Santiago, 3810 Aveiro, Portugal (alex@det.ua.pt).
}

(SPC) is one of the simplest and most often used strategies to compensate time delay systems. In this algorithm it is important to choose the right model representation of the linear/non-linear system. The model should be accurate and robust for all working points, with a simple mathematical and transparent representation that makes it interpretable.

This work is based in a previews study made in modelling and controlling a gas water heater system. The problem was to control the output water temperature even with water flow, cold water temperature and desired hot water temperature changes. To succeed in this mission one non-linear model based Smith predictive controller was implemented. The main study was to identify the best and simple model of the gas water heater system.

It has been shown that many variable industry linear and nonlinear processes are effectively modelled with neural and neuro-fuzzy models like the chemical processes [1]. Hammerstein and Wiener models like $\mathrm{pH}$-neutralization, heat exchangers and distillation columns [2]-[3]. And hybrid models like heating and cooling processes, fermentation [4], solid drying processes [5] and continues stirred tank reactor (CSTR) [6].

In this previews work there were explored this three different modelling types: neuro-fuzzy [7], Hammerstein [8] and hybrid [9] and [10] models that reflex the evolution of the knowledge about the first principles of the system. These kinds of models were used because the system had a non-linear actuator and time varying linear parameters.

At the beginning there was no knowledge about the physical model and there were used black and grey box model approaches. Finally, the physical model was found and a much simple adaptive model was achieved (the physical model white box modelling).

This paper presents two different control algorithms to control the output water temperature in an electric water heater system. The first approach is the adaptive proportional integral derivative controller and second is the Smith predictive controller based on the physical model of the system. From the previews work it is known that the first control approach is not the best algorithm to use in this system, it was used just because it has a simple mathematical structure and serves to compare results with the Smith predictive controller results. The Smith predictive controller has a much more complex mathematical structure because it uses three internal physical models (one inverse and two directs) and deals with the variable time delay of the system. The knowledge of the physical model permits varying the linear parameters correctly 
in time and gives an interpretable model that facilitate its integration on any control schemes.

This paper starts, in section II, with a full description of the implemented system to control the electric water heater, including a detailed description of the heater and its physical equations allowing the reader to have a comprehension of the control problems that will be explained in later sections.

Section III and IV, describes the two control algorithms presented: the adaptive proportional integral derivative control structure and the Smith predictive control based in the physical models of the heater. These sections show the control results using the two approaches applied in to a domestic electric water heater system.

Finally, in section $\mathrm{V}$, the conclusions and future works are presented.

\section{THE ELECTRIC WATER HEATER}

The overall system has three main blocks: the electric water heater, a micro-controller board and a personal computer (see figure 1).

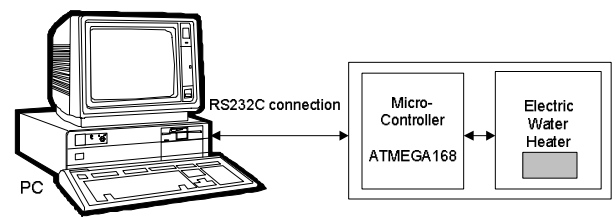

Fig. 1. System main blocks.

The micro-controller board has two modules controlled by a flash-type micro-controller from the ATMEL, ATMEGA168 with 8 Kbytes on FLASH. The interface module has the necessary electronics to connect the sensors and control the actuator. The communication module has the RS232 interface used for monitoring and acquisition of all system variables in to a personal computer.

After this small description of the prototype system, the electric water heater characteristics are presented and its first principles equations are presented.

\section{A. Electric Water Heater Description}

The electric water heater is a multiple input single output (MISO) system. The controlled output water temperature will be called hot water temperature $(h w t(t))$. This variable depends of the cold water temperature $(c w t(t))$, water flow $(w f(t))$, power $(p(t))$ and of the electric water heater dynamics. The hot and cold water temperature difference is called delta water temperature $(\Delta t(t))$.

The electric water heater is physically composed by an electric resistance, a permutation chamber and several sensors used for control and security of the system as shown on figure 2 .

Operating range of the $h w t(t)$ is from 20 to $50^{\circ} \mathrm{C}$. Operating range of the $c w t(t)$ is from 5 to $25^{\circ} \mathrm{C}$. Operating range of the $w f(t)$ is from 0,5 to 2,5 litters / minute. Operating range of the $p(t)$ is from 0 to $100 \%$ of the available power.

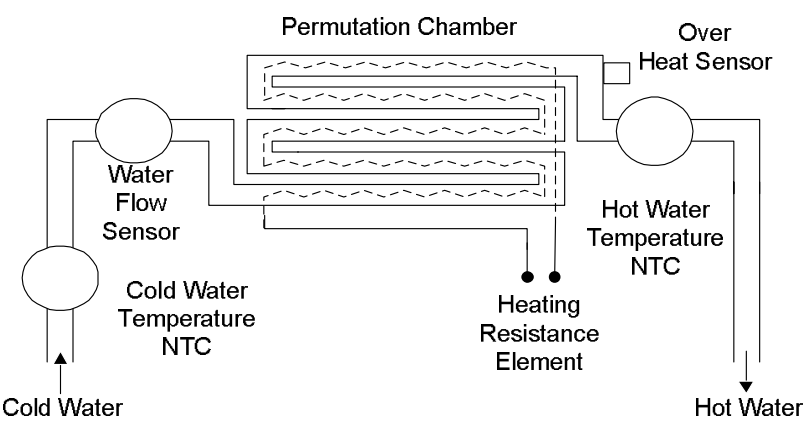

Fig. 2. Schematic of the electric water heater: sensors and actuator.

The applied energy in to the heating resistance is controlled using 100 alternated voltage cycles (one second). In each iteration, the applied number of cycles is proportional to the delivery energy to the heating element.

Figure 3 shows one photo of the electric water heater and the micro-controller board.

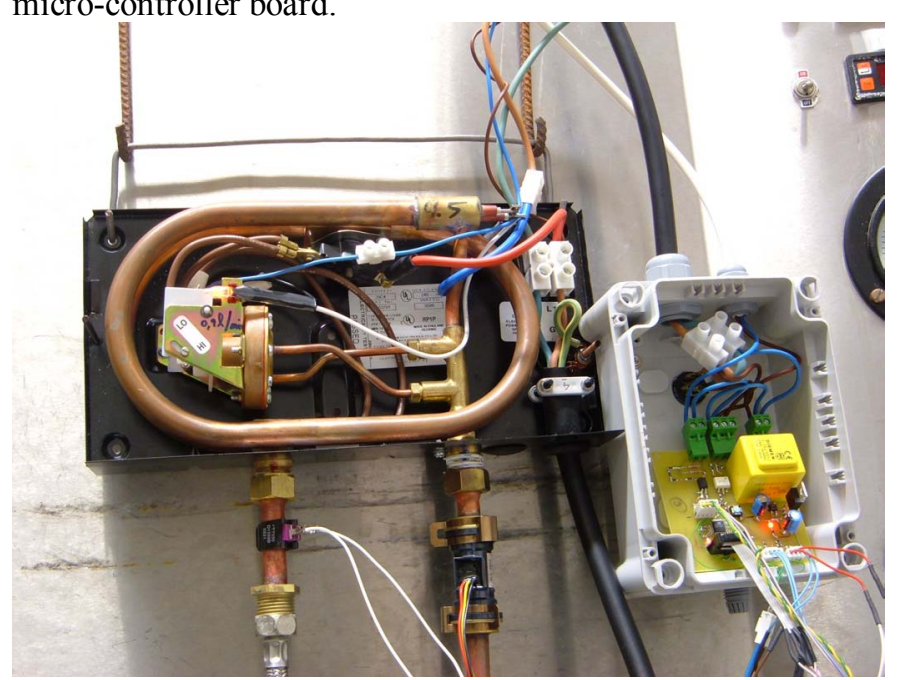

Fig. 3. Photo of the electric water heater and the micro-controller board.

\section{B. Electric Water Heater First Principles Equations}

Applying the principle of energy conservation in the electric water heater system, equation 1 could be written. This equation was based in a previews work made in modelling a gas water heater system, first time presented in [11].

$$
\frac{d E s(t)}{d t}=Q e(t-t d)-w f(t) h w t(t) C e-w f(t) c w t(t) C e
$$

Where $d E s(t) / d t=M C e(d \Delta t(t) / d t)$ is the energy variation of the system in the instant $t, Q e(t)$ is the calorific absorbed energy, $w f(t) c w t(t) C e$ is the input water energy that enters in the system, $w f(t) h w t(t) C e$ is the output water energy that leaves the system, and $C e$ is the specific heat of the water, $M$ is the water mass inside of the permutation chamber and $t d$ is the variable system time delay.

The time delay of the system has two parts: a fixed one that became from the transformation of energy and a variable part that became from the water flow that circulates in the permutation chamber. 
$M$ is the mass of water inside of the permutation chamber (measured value of $0,09 \mathrm{Kg}$ ) and $\mathrm{Ce}$ is the specific heat of the water (tabled value of $4186 \mathrm{~J} /(\mathrm{KgK})$ ). The maximum calorific absorbed energy $Q e(t)$ is proportional to the maximum electric applied power of 5,0 $\mathrm{KW}$.

The absorbed energy $Q e(t)$ is proportional to the applied electric power $p(t)$. On each utilization of the water heater it was considered that $c w t(t)$ is constant, it could change from utilization to utilization, but in each utilization it remains approximately constant. Its dynamics does not affect the dynamics of the output energy variation because its variation is too slow.

Writing equation 1 in to the Laplace domain and considering a fixed water flow $w f(t)=W f$ and fixed time delay $t d$, it gives equation 2 .

$$
\frac{\Delta t(s)}{Q e(s)}=\frac{\frac{1}{W f C e}}{\frac{M}{W f} s+1} e^{-s t d}=\frac{\frac{1}{W f C e} \frac{W f}{M}}{s+\frac{W f}{M}} e^{-s t d}
$$

Passing to the discrete domain, with a sampling period of $h=1$ second and with discrete time delay $\tau d(k)=\operatorname{int}\left(\frac{t d(t)}{h}\right)+1$, the final discrete transfer function is illustrated in equation 3.

$$
\Delta t(k+1)=\left(e^{-\frac{W f}{M}}\right) \Delta t(k)+\left(\frac{1}{W f C e}\left(1-e^{-\frac{W f}{M}}\right)\right)(\operatorname{Qe}(k-\tau d(k)))
$$

The real discrete time delay $\tau d(k)=\tau d_{1}(k)+\tau d_{2}(k)$ is given in equation 4 , where $\tau d_{1}(k)=3 s$ is the fixed part of $\tau d(k)$ that became from the transformation of energy and $\tau d_{2}(k)$ is the variable part of $\tau d(k)$ that became from the water flow $w f(k)$ that circulates in the permutation chamber.

$$
\tau d(k)=\left\{\begin{array}{lll}
4 & \text { to } & w f(k)>=1,75 l / \mathrm{min} \\
5 & \text { to } & 1,00 \mathrm{l} / \mathrm{min}<w f(k)<1,75 \mathrm{l} / \mathrm{min} \\
6 & \text { to } & w f(k)<=1,00 \mathrm{l} / \mathrm{min}
\end{array}\right.
$$

Considering now the possibility of changes in the water flow, in the discrete domain $W f=w f(k)$ and $\tau d_{2}(k)$, the final transfer function is given in equation 5 .

$$
\begin{aligned}
& \Delta t(k+1)=\left(e^{-\frac{w f\left(k-\tau d_{2}(k)\right)}{M}}\right) \Delta t(k)+ \\
& \left(\frac{1}{w f\left(k-\tau d_{2}(k)\right) C e}\left(1-e^{-\frac{w f\left(k-\tau d_{2}(k)\right)}{M}}\right)\right)(\operatorname{Qe}(k-\tau d(k)))
\end{aligned}
$$

Observing the real data of the system, the absorbed energy $Q e(t)$ is a linear static function $f($.) proportional to the applied electric power $p(t)$ as expressed in equation 6 .

$Q e(k-\tau d(k))=f(p(k-\tau d(k)))$

Finally, the discrete global transfer function is given by equation 7 .

$$
\begin{aligned}
& \Delta t(k+1)=\left(e^{-\frac{w f\left(k-\tau d_{2}(k)\right)}{M}}\right) \Delta t(k)+ \\
& \left(\frac{1}{w f\left(k-\tau d_{2}(k)\right) C e}\left(1-e^{-\frac{w f\left(k-\tau d_{2}(k)\right)}{M}}\right)\right)(f(p(k-\tau d(k))))
\end{aligned}
$$

If $A(k)$ and $B(k)$ are defined as expressed in equation 8 , the final discrete transfer function is given as defined in equation 9.

$$
\begin{aligned}
& A(k)=e^{-\frac{w f\left(k-\tau d_{2}(k)\right)}{M}} \\
& B(k)=\frac{1}{w f\left(k-\tau d_{2}(k)\right) C e}\left(1-e^{-\frac{w f\left(k-\tau d_{2}(k)\right)}{M}}\right) \\
& \Delta t(k+1)=A(k) \Delta t(k)+B(k)(f(p(k-\tau d(k))))
\end{aligned}
$$

\section{Physical Model Validation}

For validation of the presented discrete physical model, it is necessary to have open loop data of the real system. This data has been chosen to respect two important requirements: frequency and amplitude spectrum wide enough [12]. Respecting the necessary presupposes, the collect data is made via RS232 connection to the PC. The validation data and the physical model error are illustrated in figure 4.

Figure 4 shows the physical model error signal $e(k)$, which is equal to the difference between delta and estimated delta water temperature $e(k)=\Delta t(k)-\Delta t_{\text {estimated }}(k)$. It can be seen from this signal, that the proposed model achieved very good results with a mean square error $(M S E)$ of $1,32^{\circ} \mathrm{C}^{2}$ for the all test set (1 to 1600$)$.

From the validation test, figure 5 shows the two linear variable parameters expressed in equation 8 of the physical model used.

As can be seen the $A(k)$ parameter that multiply with the regressor delta water temperature $\Delta t(k)$ changes significantly with water flow $w f(k)$ and the $B(k)$ parameter that multiply with the regressor applied power $f(p(k-\tau d(k)))$ presents very small changes with the water flow $w f(k)$. 

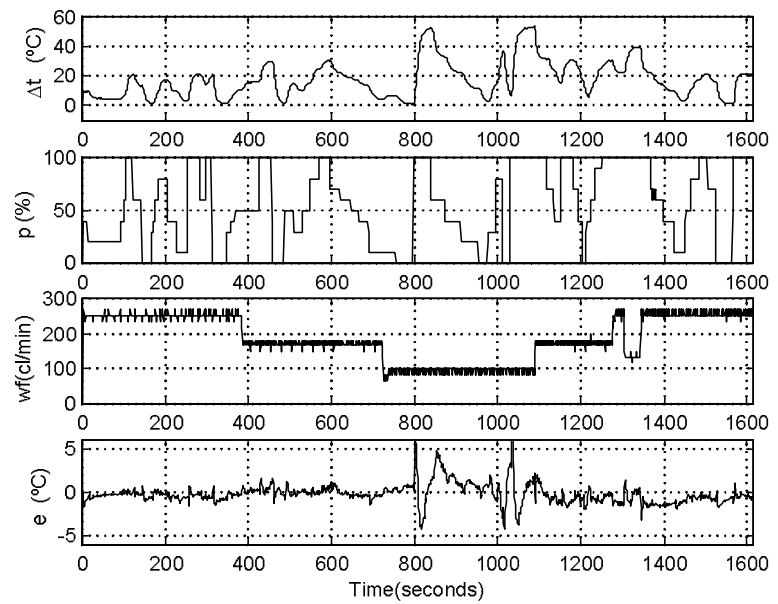

Fig. 4. Open loop data used to validate the model.

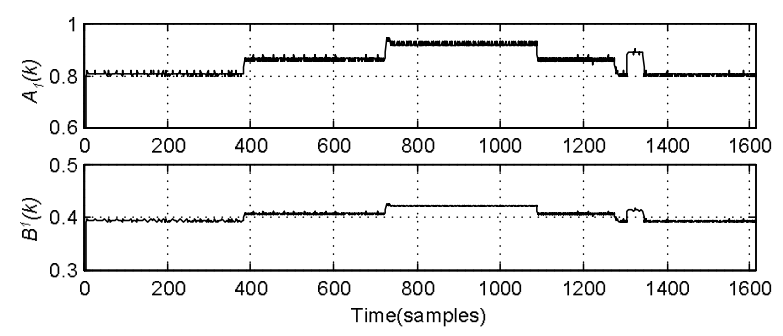

Fig. 5. The two linear variable parameters $A(k)$ and $B(k)$.

From the results it can be seen that for the small water flows the model presents a bigger error signal. This happens because of the small resolution of the water flow measurements and of the estimated integer time delays forced (a multiple of the sampling time $h$ it is not possible fractional time delays).

\section{ADAPTIVE PID CONTROLLER}

The first control loop tested is the adaptive proportional integral derivative control algorithm. Adaptive because we know that gain and time constant of the system changes with the input water flow. First it is described the control structure and its parameters and second the real control results are showed.

\section{A. Adaptive PID Control Structure}

This is a very simple and well known control strategy that has two control parameters $K_{p}$ and $K_{d}$ that are multiplied by the water flow, as illustrated in figure 6 . The applied control signal $f(p(k))$ is expressed in equation 10 :

$$
\begin{aligned}
& f(p(k))=f(p(k-1))+w f(k) K_{p} e(k) \\
& +w f(k) K_{d}(e(k)-e(k-1))
\end{aligned}
$$

The $P$ block gives the error proportional contribution, the $D$ block gives the error derivative contribution and the $I$ block gives the control signal integral contribution.

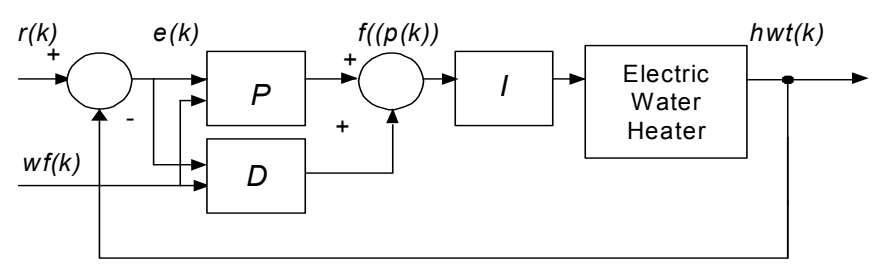

Fig. 6: APID controller constituent blocks.

The three control parameters were adjusted after several experimental tests in controlling the real system. This algorithm has some problems dealing with time constant and time delay variations of the system. With this control loop it is not possible to define a close loop system with a fixed time constant. The time delay is also a problem that is not solved with this control algorithm.

It was define a reference signal $r(t)$ that is the desired hot water temperature and a water flow $w f(t)$ with several step variations similar to the ones used in real applications. The cold water temperature was almost constant around $13,0^{\circ} \mathrm{C}$.

For testing the controllers it can be seen that error signal $e(t)=r(t)-h w t(t)$ is around zero excepted in the input transitions. In reference step variations it can be seen that the overshoots for the different water flows are similar but the rise times are clearly different, for small water flows the controller presets bigger rise times. In water flow variations the control loop have some problems because of the variable time delay. This control loop only reacted when error appears.

\section{B. Adaptive PID Control Results}

With the proposed tests signals, the tuned adaptive PID control structure was tested in controlling the electric water heater. The APID control results are shown in figure 7.

As it was predicted the results have shown some problems in water flow variations because the controller just reacts when it feels an error signal different from zero.
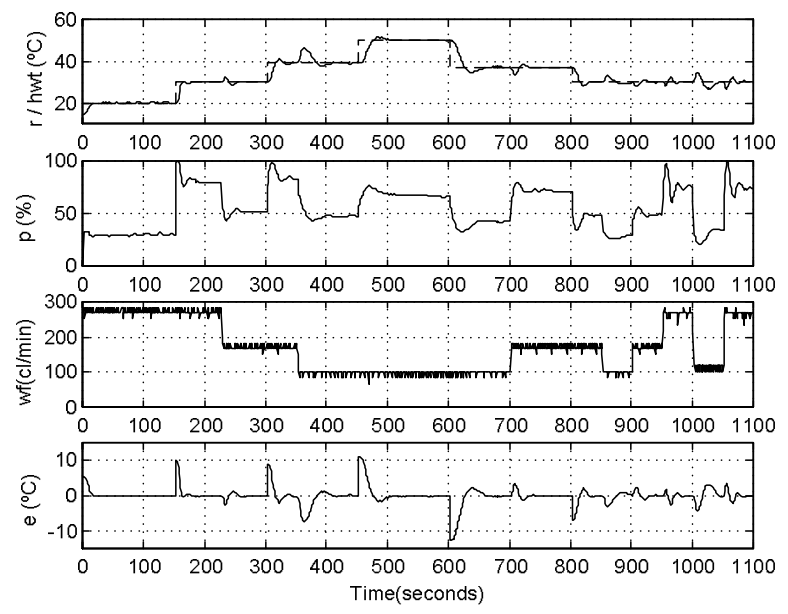

Fig. 7. Adaptive PID control results. 
The evaluation control criterion used is the mean square error (MSE). The MSE in the all test is presented in table I.

TABLE I.

MeAn SQUare ERrors OF THE CONTROL RESUltS.

\begin{tabular}{lll}
\hline Algorithm & MSE & Test Set \\
\hline APID & 5,97 \\
\hline
\end{tabular}

\section{SMITH PREDICTIVE CONTROLLER}

The second control loop tested is the Smith predictive control algorithm. This control strategy is particularly used to control systems with time delay. First it is described the control structure and its parameters and second the control results are showed.

\section{A. Smith Predictive Control Structure}

The Smith predictive controller is based in the internal model controller architecture that uses the physical model presented in section II, as illustrated in figure 8. It uses two physical direct models one with time delay for the prediction loop and another with out the time delay for the internal model control structure.

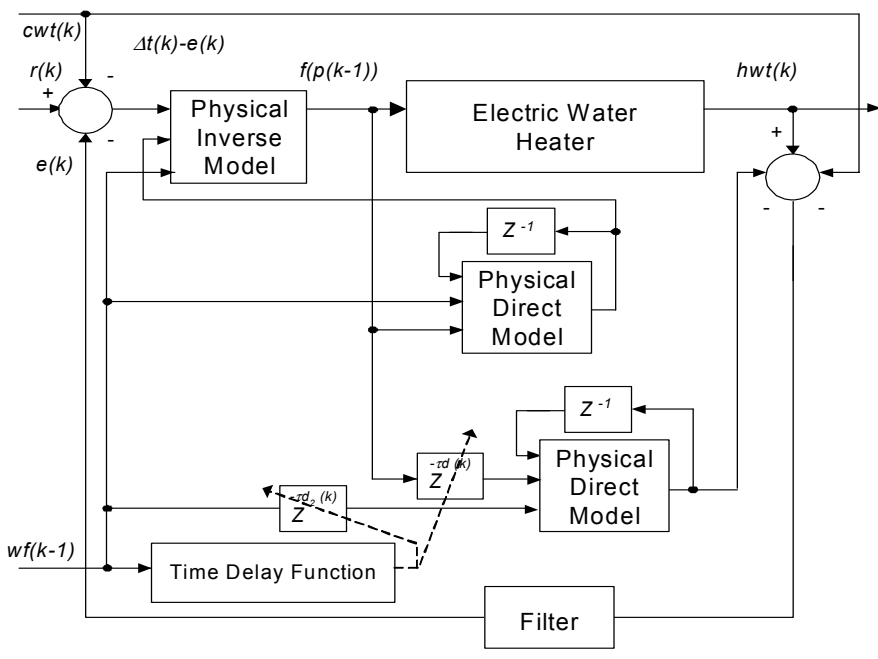

Fig. 8. SPC constituent blocks.

The Smith predictive control structure has a special configuration, because the systems has two inputs with two deferent time delays so it uses two direct models, one model with time delay for compensate its negative effect and another with out time delay needed for the internal model control structure.

The SPC separates the time delay of the plant from time delay of the model, so it is possible to predict the $\Delta t(k) \tau d(k)$ steps earlier, avoiding the negative effect of the time-delay in the control results.
The time delay is a known function that depends of the water flow $w f(k)$. The incorrect prediction of the time delay may lead to aggressive control if the time delay is under estimated or conservative control if the time delay is over estimated [13][14].

The physical inverse model is mathematically calculated based in the physical direct model presented in section II used with out time delay.

The low pass filter used in the error feedback loop is a digital first order filter used to filter the feedback error and indirectly to filter the control signal $f(p(k))$. The time delay function is a function of the water flow, which is explained in section II and expressed in equation 4.

To test the SPC based in the physical model it was used the same reference signals $r(t)$ and water flow $w f(t)$ used to test the adaptive PID controller.

\section{B. Smith Predictive Control Results}

The SPC results are shown in figure 9. As it was predicted from previews work the results are very good in reference and in water flow changes. The behaviour of the closed loop system is very similar in every working point.

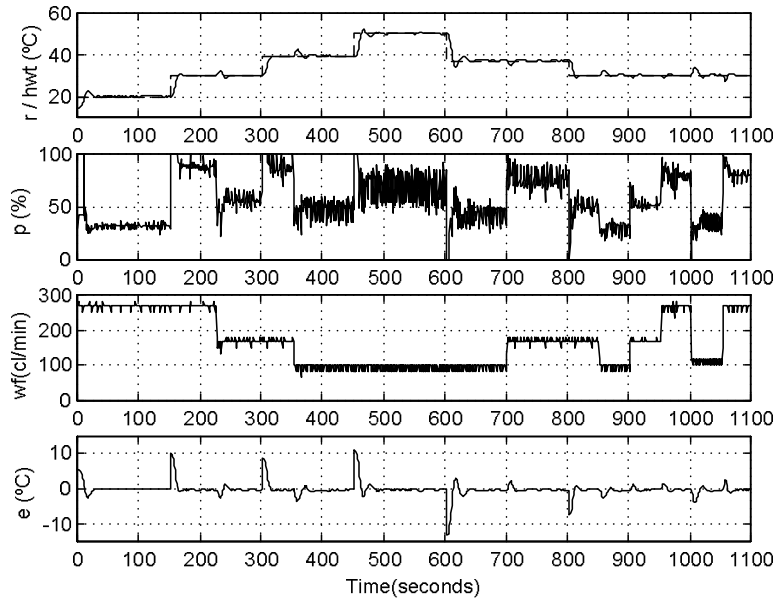

Fig. 9. SPC control results.

It can be seen that for small water flows the resolution of the measure is small that makes the control signal a bit aggressive but it does not affect the output hot water temperature.

For small water flows there is another problem with the multiplicity of the time delay and its resolution. With a sampling period of 1 second it is more difficult to use factional time delays that happen in reality. This makes the control results a bit aggressive.

The final MSE evaluation control criterion achieved with the SPC is presented in table II.

TABLE II.

MEAN SQUARE ERRORS OF THE CONTROL RESUlTS

\begin{tabular}{lll}
\hline Algorithm & MSE Test Set \\
\hline SPC & 3,56 \\
\hline
\end{tabular}


The physical model includes à priori knowledge of the real system and has the advantage of been interpretable. This characteristic facilitates the implementation and simplicity the Smith predictive control algorithm.

\section{CONCLUSIONS AND FUTURE WORK}

For comparing the two control algorithms, APID and SPC, the reference signals were applied in controlling the system and the respective mean square errors were calculated. The final results are expressed in table III.

TABLE III.

MeAN SQuare ERrors Of THE CONTROL Results.

\begin{tabular}{lll}
\hline Algorithm & MSE & Test Set \\
\hline APID & 5,97 & \\
\hline SPC & 3,56 & \\
\hline
\end{tabular}

This work present and validate the physical model of the electric water heater. This model was based in the model of a gas water heater because of the similarities of both processes.

The MSE of the validation test is very small which validate the physical electric water heater model accuracy.

Finally, the proposed APID and SPC controllers were successful applied in the electric water heater system. It is verify that the SPC achieved much better results than the adaptive proportional integral derivative controller did as it was expected because of the system characteristics.

The best control structure for varying first order systems with varying large time delay is the Smith predictive controller based in physical model of the system as presented in this work. The SPC controller proposed in opposition to the APID controller reacts also very well in cold water temperature variations.

This controller is mathematically simple and easily implemented in a microcontroller with reduce resources.

For future work some improvements should be made as the enlargement of the resolution of the used water flow and the redefinition of the time delay function.

\section{REFERENCES}

[1] M. L. Tompson. And M. A. Kramer, "Modelling chemical processes using prior knowledge and neural networks", A. I. Ch. E. Journal, 1994, vol. 40(8), pp. 1328-1340.

[2] M. Pottman, R. K. Pearson, "Block-Oriented NARMAX Models with Output Multiplicities", AIChE Journal, 1998, vol. 44(1), pp. 131-140.

[3] E. Eskinat, S. H. Johnson and W. Luyben, "Use of Hammerstein Models in Identification of Non-Linear Systems", AIChE Journal, 1991, vol. 37(2), pp. 255-268.

[4] D. C. Psichogios and L. H. Ungar, "A hybrid neural network-first principles approach to process modelling", AIChE Journal, 1992, vol. 38(10), pp. 1499-1511.

[5] F. A. Cubillos, P. I. Alvarez, J. C. Pinto, E. L. Lima. "Hybrid-neural modelling for particulate solid drying processes". Power Thecnology, 1996, vol. 87, pp. 153-160.

[6] J. Abonyi, J. Madar and F. Szeifert, "Combining First Principles Models and Neural Networks for Generic Model Control", Soft Computing in Industrial Applications - Recent Advances, Eds. R. Roy, M. Koppen, S. O., T. F., F. Homann Springer Engineering Series, 2002, pp.111-122.
[7] J. Vieira, A. Mota - "Smith Predictor Based Neural-Fuzzy Controller Applied in a Water Gas Heater that Presents a Large Time-Delay and Load Disturbances", Proceedings IEEE International Conference on Control Applications, Istanbul, Turkey, 23 a 25 June 2003, vol. 1, pp. 362-367.

[8] J. Vieira, A. Mota - " Parameter Estimation of Non-Linear Systems With Hammerstein Models Using Neuro-Fuzzy and Polynomial Approximation Approaches", Proceedings of IEEE-FUZZ International Conference on Fuzzy Systems, Budapest, Hungary, 25 a 29 July 2004, vol. 2, pp. 849-854.

[9] J. Vieira, F. Dias and A. Mota, "Hybrid Neuro-Fuzzy Network-Priori Knowledge Model in Temperature Control of a Gas Water Heater System", Proceedings of $5^{\text {th }}$ International Conference on Hybrid, Intelligent Systems, Rio de Janeiro, 2005.

[10] J. Vieira.,A. Mota, 2004a. Water Gas Heater Non-Linear Physical Model: Optimization with Genetic Algorithms. Proceedings of IASTED 23rd International Conference on Modelling, Identification and Control, February 23-25, vol. 1, pp. 122-127.

[11] J. Vieira, A. Mota, "Water Gas Heater Non-Linear Physical Model: Optimization with Genetic Algorithms", Proceedings of IASTED $23^{\text {rd }}$ International Conference on Modelling, Identification and Control, 2004.

[12] D. C. Psichogios and L. H. Ungar, "A hybrid neural network-first principles approach to process modelling", AIChE Journal, 1992, vol. 38(10), pp. 1499-1511.

[13] Y. Tan, M. Nazmul Karim, "Smith Predictor Based Neural Controller with Time Delay Estimation", Proceedings of 15th Triennial World Congress, IFAC, 2002

[14] Y. Tan, A. R. Van Cauwenberghe, "Neural-network-Based d-step-ahead Predictors for Nonlinear Systems with Time Delay", Engineering Applications of Artificial Intelligence, 1999, vol. 12(1), pp. 21-35. 